\title{
Centro de Emprendimiento e Innovación. Una estrategia de desarrollo institucional
}

\section{Entrepreneurship and Innovation Center. An Institutional Development Strategy}

\author{
Rafael Méndez Lozano \\ Director Centro de Emprendimiento e Innovación \\ Universidad Surcolombiana
}

\section{Origen de la idea}

El origen de la idea de crear el Centro de Emprendimiento e Innovación CEIN, se remonta a una propuesta de investigación del Grupo e Investigación CREA de la Facultad de Economía y Administración de la Universidad Surcolombiana, de disponer de un sitio donde miembros de la comunidad universitaria interesados en desarrollar una iniciativa social, cultural deportiva, ambiental o empresarial pudieran contar con la asesoría y acompañamiento para desarrollar la idea y estructurar el proyecto.

Como resultado del proyecto de investigación que incluyó un análisis sobre las razones de orden nacional, regional e institucional que motivaban esta propuesta, así como el estudio del marco legal y normativo existente y del análisis de factores de éxito y limitaciones en instituciones universitarias públicas y privadas del país a partir de una labor de campo, se concluyó en una propuesta a la medida de institución que se materializó en la publicación denominada "Emprendimiento. Una estrategia de desarrollo institucional" y fue un referente para instituciones universitarias.

Como factores de éxito de una Unidad o Centro de Emprendimiento, se identificaron los siguientes:

- De orden académico

$\checkmark$ Definición un modelo y una concepción filosófica de la innovación y el emprendimiento.

$\checkmark$ Definición de criterios y metodologías de trabajo.

$\checkmark$ Incluir diversos tipos de emprendimiento: social, cultural deportivo ambiental, empresarial.

$\checkmark$ Articulación de la investigación con la innovación y el emprendimiento.

$\checkmark$ Fomento de equipos de trabajo interdisciplinarios.

$\checkmark$ Creación de un centro de documentación en emprendimiento.

$\checkmark$ Priorización de la investigación aplicada que conduzcan a propuestas de innovación.

$\checkmark$ Elaboración de un portafolio de servicios.

- De orden organizativo

$\checkmark$ Precisar la población objetivo.

$\checkmark$ Ubicación estratégica del Centro o Unidad en la estructura organizativa de la institución.

$\checkmark$ Disposición y actitud de servicio de los docentes a los investigadores y emprendedores.

$\checkmark$ Articulación del Centro de Emprendimiento con unidades de apoyo internas de la institución: consultorios, laboratorios, emisora entre otros.

- De orden organizativo

$\checkmark$ Articulación del Centro con la Red Departamental del Emprendimiento $y$ redes nacionales $e$ internacionales.

$\checkmark \quad$ Integración del emprendimiento con la proyección social

- De orden económico

$\checkmark$ Gestionar presupuesto institucional para el emprendimiento.

$\checkmark$ Acercamiento de los egresados como actor importante de apoyo.

$\checkmark$ Establecimiento de relaciones con el sector productivo.

$\checkmark \quad$ No olvidar que el estudiante es el actor principal y se pretende es cambiar formas de pensar.

$\checkmark$ Crear un sistema de medición y estímulos.

\section{Creación oficial}

El Centro de Emprendimiento e Innovación CEIN de la Universidad Surcolombiana oficialmente fue creado por el Consejo Superior Universitario, mediante Acuerdo 004 de enero de 2015, con la misión de "Promover y gestionar la cultura del emprendimiento y la innovación en la comunidad, a partir de la identificación y estructuración de ideas y proyectos de creación y fortalecimiento de iniciativas emprendedoras de carácter social, cultural deportivo, empresarial, que conjuguen con un enfoque sistémico la investigación, el conocimiento, la interdisciplinariedad, la innovación y el emprendimiento de alto impacto para el crecimiento económico y social".

\section{Usuarios y servicios}

Como usuarios del CEIN se han identificado los siguientes grupos de interés: estudiantes de pregrado y postgrado, grupos y semilleros de investigación, 
docentes, empleados y trabajadores, egresados, padres de familia y empresarios.

Los servicios que ofrece el CEIN se agrupan en tres categorías: Conceptualización de la innovación y el emprendimiento, asesoría y acompañamiento y aceleración.

En la categoría de conceptualización de la innovación y emprendimiento, el énfasis se hace en el apoyo a los procesos formativos de los estudiantes; en tal sentido, se asesora a los diferentes programas académicos en el diseño de propuestas curriculares asociadas a estas temáticas y se ofrecen cursos y talleres en creatividad, emprendimiento, innovación, estructuración de proyectos, liderazgo, trabajo en equipo e historia empresarial.

En la categoría de asesoría y acompañamiento, se pretende brindar a los miembros de la comunidad universitaria que lleguen al CEIN con una idea de emprendimiento, la asesoría y seguimiento en el proceso de consolidación de sus ideas, estructuración técnica de proyectos y planes de empresa y se orientan en contactos institucionales y en la participación en concursos regionales, nacionales e internacionales.

Los servicios de la categoría de aceleración, va dirigida principalmente a las PYMES con el propósito de fortalecer la relación Estado-Sector ProductivoAcademia, mediantes la oferta de diplomados de actualización que incluyen las temáticas de innovación, mercados, finanzas, procesos de producción, talento humano y gerencia empresarial. Esta relación permitirá simultáneamente, identificar necesidades del sector real para focalizar la investigación aplicada y la consultoría desde la Universidad.

\section{Idea, construye, transforma}

El eslogan que refleja la filosofía del CEIN se define como idea, construye, transforma, como un reconocimiento a la creatividad y capacidad del ser humano para generar ideas en las diferentes áreas del conocimiento, el construye se relaciona con la necesidad de aplicar metodologías pertinentes para estructurar con éxito un proyecto, como un requisito para tomar decisiones con menos nivel de incertidumbre y riesgo y transforma es la razón de ser de los procesos de innovación y emprendimiento, pues además de transformar la idea y la mentalidad de los miembros del equipo gestor del proyecto, la puesta en operación o funcionamiento de proyectos asociados al conocimiento, transforma positivamente la sociedad cuando se disminuye la informalidad $y$ ofrecen mejores productos/servicios, nuevas fuentes de empleo y mejor remuneradas, mayor articulación de la investigación con la innovación y el emprendimiento y se genera un mayor impacto regional y nacional.

\section{Ecosistema interno y externo}

Para alcanzar el objetivo de articular la innovación y el emprendimiento con los tres ejes misionales de la Universidad, es decir, la docencia, la investigación y la proyección social, se concibe el CEIN como un catalizador de esos ejes misionales y la mejor forma de alcanzarlo, es apuntándole a la construcción de un ECOSISTEMA INTERNO, que consiste básicamente en articular acciones relacionadas con la investigación aplicada, la innovación y el emprendimiento con los programas académicos tanto de pregrado como de postgrado, con los semilleros y grupos de investigación y con otras unidades de apoyo como los laboratorios, consultorios jurídico y psicológico, la emisora universitaria, las asociaciones de estudiantes y profesores entre otros. De esta manera cuando la comunidad universitaria comparta conceptos y se construya un imaginario colectivo que evidencie que si es posible aplicar el conocimiento desde las diferentes áreas del conocimiento para el desarrollo iniciativas emprendedoras de alto impacto, la Universidad obtiene nuevos retornos en reconocimiento social por el aporte de su comunidad y egresados en el desarrollo de proyectos de interés regional y nacional, por la imagen corporativa y por el interés de otros actores externos de trabajar de la mano con nuestra institución en beneficio de la sociedad.

Así como se debe construir comunidad con mentalidad de aplicar el conocimiento en propuestas y soluciones creativas útiles para los emprendedores y la sociedad, es necesario que la Universidad se articule con otras instituciones de la cadena de valor de la innovación y el emprendimiento como Cámara de Comercio, SENA, entidades de financiación, centros tecnológicos, instituciones públicas y privadas de apoyo al emprendimiento de orden regional, nacional e internacional, gremios y otras instituciones de educación superior. La acción conjunta y colaborativa de estas instituciones, en la que cada una cumple su labor misional y trabajando en red, es lo que denomina ECOSISTEMA EXTERNO, y es esta una condición fundamental, para jalonar de manera más eficiente y efectiva, procesos de transformación regional $y$ nacional que benefician la sociedad en su conjunto en los ámbitos social, económico y ambiental.

\section{Gestión del CEIN}

Como resultado de la gestión realizada por el CEIN, teniendo en cuenta que mediante el acuerdo 004 de enero de 2015 se adoptó la innovación y el emprendimiento como una política institucional y que la inauguración del sitio de atención del CEIN a la comunidad se realizó el día 11 de agosto de 2015, los logros alcanzados son los siguientes: 
- Adecuación espacio físico del CEIN en la Facultad de Economía.

- "Mobiliario, dotación y construcción de jardines verticales construidos por estudiantes y egresados empresarios de la comunidad universitaria.

- Conformación equipo base con la vinculación de tres egresados y seis monitores de diferentes programas académicos.

- Reconocimiento del CEIN a un egresado empresario destacado German Antonio Melo.

- Convocatoria al acto de Inauguración del CEIN de 150 participantes conformados por empresarios, sector público y miembros de la comunidad universitaria.

- Atención a visita de 5 pares académicos de programas nuevos de pregrado y postgrado.

- Establecimiento de relaciones de intercambio académico y de experiencias en emprendimiento con la Unidad de Emprendimiento de la Universidad Nacional de Colombia.

- Asesoría a 46 personas y equipos de trabajo de la comunidad universitaria. ( $76 \%$ hombres y $24 \%$ mujeres).

- Realización de eventos de sensibilización sobre innovación y emprendimiento con participación registrada de 358 miembros de la comunidad universitaria.

- Participación en reuniones de trabajo en emprendimiento, convocadas por la Cámara de Comercio de Neiva, SENA, Red de Emprendimiento "Huila Emprende", Micitio, Gobernación del Huila y Ministerio de Industria, Comercio y Turismo.

- Representación de la Universidad como conferencista en "Creatividad e Innovación en Emprendimiento" en la Universidad Mariana de Pasto.

- Participación con jóvenes emprendedores con prototipos en la Feria del Emprendimiento de la Universidad de Ibagué.

- Publicación del proyecto de investigación "Diagnóstico, factores críticos y lecciones de aprendizaje de las iniciativas empresariales financiadas por el Fondo Emprender en el Huila. Período 2002-2014.

- Visita de tres miembros del equipo de trabajo del CEIN a la Universidad Nacional de Colombia para transferencia de metodologías, experiencias de la Unidad de Emprendimiento.

- Sustentación ante pares académicos del Ministerio de Educación Nacional del proyecto de creación de la Maestría en Gerencia Integral de Proyectos.

- Articulación con la comunidad universitaria a través de redes sociales. A la fecha se cuenta con 506 seguidores.

- Establecimiento de contactos del CEIN con el Centro de Informática y Biología Computacional BIOS de la ciudad de Manizales y la Universidad de Caldas como actor líder de Manizales Más.

Con la creación del CEIN, se inician procesos de transformación institucional que dejan en evidencia que si es posible articular la docencia con la investigación, la innovación y el emprendimiento. 